\title{
Lexicometría: enfoque aplicado a la redefinición de conceptos e identificación de unidades temáticas
}

\author{
Ivón Romero-Pérez \\ Yolima Alarcón-Vásquez \\ Rafael García-Jiménez \\ Universidad Simón Bolívar - UNISIMON, Colombia
}

ORIGINAL

\begin{abstract}
Resumen
Objetivo. Redefinir la expresión conceptual Proyecto Pedagógico Productivo (PPP) e identificar los enfoques temáticos derivados de la extracción de patrones de conocimiento inherentes en la descomposición y comparación de unidades léxica derivadas de varias definiciones aplicando el análisis lexicométrico.

Método. Este estudio es de naturaleza documental con alcance descriptivo, se desarrolla con la aplicación del método lexicometrico o de análisis textual.Se determinó un muestreo intencional y no probabilístico, con una población constituida por todos los documentos sobre el tema disponible en google scholar y bases de datos bibliográficas.

Resultados. Se identificaron las unidades léxicas: rural, vida, familia y social, con un alto grado de distribución en comparación con docentes, estudiantes, estrategia y aprendizaje, que emergen de manera preponderante en las distintas representaciones gráficas derivadas del análisis de correspondencia.

Conclusiones. La lexicometría una herramienta que contribuyen a la extracción de conocimiento relevante para la redefinición de un concepto desde el punto de vista de su representación colectiva, sin embargo, para la identificación de las categorias de análisis éstos deben complementarse con la revisión documental, a fin de extraer del legado historico y del estado del arte, las unidades temáticas que sólo emergen de un análisis cualatitativo.
\end{abstract}

Palabras-clave

Análisis de correspondencia; Análisis textual; Corpus; Lexicometría; Proyectos pedagógicos productivos

\section{Lexicometry and textual analysis applied to the identification of thematic approaches and redefinition of concepts}

\begin{abstract}
Objective. Redefine the conceptual expression productive pedagogical project (PPP) and identify thematic approaches derived from the extraction of knowledge patterns inherent in the decomposition and comparison of lexical units derived from several definitions.

Method. This study is of documentary nature with descriptive scope, it is developed with the application of lexicometric methods and textual analysis. It was determined an intentional and non-probabilistic sampling, with a population constituted by all the documents on the subject available in google scholar and databases Bibliographical references.

Results. The lexical units were identified: rural, life, family and social, with a high degree of distribution in comparison to teachers, students, strategy and learning, which emerge predominantly in the different graphical representations derived from correspondence analysis.

Conclusions. Lexicometry and textual analysis are qualitative approaches that contribute to the extraction of knowledge relevant to the redefinition of a concept from the point of view of its collective representation, however, for the identific ation of the categories of analysis, these should be complemented by the documentary revision, In order to extract from the historical legacy and state of the art, the thematic units that only emerge from a qualitative analysis.
\end{abstract}

Keywords

Corpus; Correspondence analysis; Lexicometry; Productive pedagogical projects; Textual analysis 


\section{Introducción}

La lexicometría es una herramienta que pueden utilizarse para redefinir expresiones conceptuales desde el punto de vista de su representación colectiva, así como, para identificar categorías o unidades temáticas derivadas de la extracción automática de patrones de conocimiento oculto en datos de naturaleza textual. El análisis de datos textuales "se caracteriza por ser un proceso secuencial donde el investigador reflexiona sobre los datos, los categoriza y los relaciona con el propósito de identificar las categorías centrales que aportan información emergente a la comprensión de un problema particular" (Barreto, Velandia-Morales, \& RincónVásquez, 2011, p. 12).

Su aplicación puede ir desde la identificación de tendencias o preferencias léxicas emergentes de un corpus, a través de la cuantificación de la ocurrencia de las palabras más significativas (Páramo, 2010; Vázquez-Cano, Mengual-Andrés \& Roig-Vila, 2015). Las inferencias de nuevas ideas y características derivadas de la descomposición y comparación de las unidades léxicas inherentes a un corpus compuesto por un conjunto de definiciones (Acosta, 2013; Barreto, Velandia-Morales, \& Rincón-Vásquez, 2011; Escalante, 2009; Césari, 2007). Asimismo, como en la identificación de categorías o unidades temáticas emergentes producto del análisis de las diferencias y semejanzas que surgen de la comparación léxica de un conjunto textos (Terrazas, 2016; Moscoloni, 2011).

Este método de análisis estadístico, a lo largo de los años han ayudado a reducir el tiempo y el esfuerzo del ser humano en el análisis de datos, todo gracias a la expansión de las nuevas tecnologías aplicadas al procesamiento automático de datos textuales, que están contribuyendo en la sistematización, comparación y categorización de la información (Satriano \& Moscoloni, 2000). El software R, es uno de ello, un lenguaje de programación de código abierto utilizado para el tratamiento estadístico de datos y la automática de los métodos de análisis empleados en la inferencia de un campo conceptual, ampliamente utilizado en la identificación de palabras, categorías y unidades temáticas emergentes dentro del tratamiento y procesamiento de datos (Baccalá, \& De la Cruz, 2000; Zamora \& Vallejos, 2012).

Desde este punto de vista, estos recursos se utilizarán para analizar y redefinir la expresión conceptual PPP, teniendo en cuenta que este concepto puede definirse como una estrategia de formación y desarrollo social, de corte pragmático, integradas a los planes de estudios a través de actividades pedagógicas y didácticas que articulan los contenidos teóricos con experiencias prácticas, los cuales, han sido adoptados principalmente por escuelas que utilizan modelos educativos flexibles que promueve el emprendimiento como alternativa de solución a problemas cotidianos de la comunidad educativa y del sector rural, ante necesidades e interés de los jóvenes por iniciar sus proyectos de vida productivos (Gélvez, 2000; Novoa, 2004; Ramírez, 2007; Ramón, 2010; Escobar, 2011; Romero-Pérez et al., 2017a).

Esta expresión conceptual tiene una naturaleza multidimensional, que encierra en sí mismo tres intencionalidades claramente definidas en el contexto histórico: primero, la dimensión de proyecto, definida por Novoa (2005) como "un conjunto de actividades interrelacionada, orientadas a la solución de un problema, con resultados significativos y previsibles en un plazo definido, mediante la aplicación de ciertos recursos, con una metodología determinada, y bajo la responsabilidad de un personal competente" (p. 66); segundo, la dimensión pedagógica, inherente a las estrategias utilizadas para "la construcción de aprendizaje significativo, el desarrollo de competencias en los estudiantes y los procesos de enseñanza que teniendo en cuenta la flexibilidad en tiempo y espacio, vinculan los aportes interdisciplinarios de diferentes agentes educativos" (Ministerio de Educación Nacional [MEN], 2011, p.11); y finalmente, la dimensión productiva relacionada con las acciones u operaciones que posibilitan la articulación de la escuela con la comunidad y el sector productivo, de tal manera, que con éstos se puedan realizar actividades productivas basada en la co-creación de productos, bienes o servicios que promuevan el emprendimiento como alternativa de solución a los problemas socioeconómicos de las zonas rurales y en condiciones vulnerables (Novoa, 2005; Romero-Pérez, 2017b).

\section{Objetivos}

El objetivo de este estudio es redefinir la expresión conceptual proyecto pedagógico productivo (PPP) e identificar los enfoques temáticos derivados de la extracción de patrones de conocimiento inherentes en la descomposición y comparación de unidades léxicas derivadas de varias definiciones aplicando el análisis lexicométrico. 


\section{Metodologia}

\subsection{Población y muestra}

La población está representada por todos los documentos sobre el tema disponible en google scholar y en bases de datos bibliográficas. Del mismo modo, se determinó un muestreo intencionado y no probabilístico, a través del cual, se seleccionaron aquellos documentos que cumplieron los siguientes criterios de inclusión y exclusión: a) documentos que describen de manera explícita el concepto, b) documentos escritos en el idioma español, c) documentos publicados en el periodo 2000 a 2014, d) documentos publicados en los formatos de texto pdf o word y f) se excluyeron blogs, páginas web y presentaciones en power point. También se tomó una muestra 17 documentos que definen de manera explícita los PPP, extrayendo de ellos 25 definiciones (segmentos de texto) con las cuales se construyó el corpus.

\subsection{Instrumento}

Él presente estudio es de tipo descriptivo multidimensional y se fundamente en el análisis de datos textuales propuesto por Lebart, Salen y Bécue (2000), quienes describen los procedimientos necesarios para la realización de un estudio "descriptivo unidimensional en el que se resumen o cuantifican las frecuencias relativas de palabras o estructuras del lenguaje y, por otra, un estudio descriptivo multidimensional en que se aplican herramientas estadísticas para analizar cuantitativamente la estructura de asociación de palabras" (Orozco, Barreto, \& Sánchez, 2008, p.282)

\subsection{Procedimiento de recogida y análisis de datos}

El método parte de un análisis descriptivo unidimensional (análisis lexicométrico) y continua con un análisis descriptivo multidimensional (Barreto, Sabucedo \& López-López, 2008). Con el primero, se obtiene un vocabulario y una nube de palabras frecuentes que representa el contexto del lenguaje; y con el segundo, se obtiene una representación gráficamente de las similitudes y asociaciones que existen entre las palabras (unidades léxicas) y las expresiones conceptuales incluidas dentro del corpus (Césari, 2007; Borja-Orozco; Rodríguez \& Alessandrini, 2014). Este procedimiento se llevó a cabo utilizando un código de programación ejecutado a través del software R, siguiendo las operaciones descritas por Bécue, M., Lebart, L., \& Rajadell, N. (1992), Césari (2007), Fernández, Modroño \& Landaluce (2007), y Silva, Hernández \& Sosa (2010), tales como:

a) Creación y depuración del corpus. Tras la elección del tema de estudio y la realización de la búsqueda, selección, revisión y análisis de los documentos, se crea un corpus con el conjunto de segmentos de textos seleccionados para muestra (Anexo 1). Posteriormente, de forma automática se eliminan los símbolos y palabras poco relevantes para el estudio, como: artículos, conjunciones, pronombres, preposiciones, números y puntuaciones.

b) Creación del vocabulario. A través de un código programación (Anexo 2), se crea autonómicamente el vocabulario del corpus, el cual se reduce al conjunto de palabras con frecuencia superior a tres (Campo, 2000).

c) Lematización del corpus. Consiste en homogenizar y sustituir las palabras que tienen significados equivalentes, por un lema (unidad léxica), cuya raíz es equivalente al conjunto de palabras seleccionadas (Bécue-Bertaut, 2009; Abascal \& Franco, 2002).

d) Matriz léxica. Es una tabla contingencia de i filas por j columnas, donde las filas tienen asignadas las palabras más frecuentes del corpus, y las columnas los segmentos de texto asociados con definiciones sobre PPP. La intersección de 42 filas (palabras) y 25 columnas (segmentos de texto ) "contienen la frecuencia con que cada una de la palabras (formas gráficas) de los vocabularios son utilizadas por los individuos de cada categoría de la variable" (Oliva, Hernández, \& Castro, 2012, p.61), es decir, el número de veces que la palabra de la fila i ha sido utilizada en el segmento de la columna j.

e) Análisis de correspondencia simple (ACS). Es una técnica de análisis multidimensional, propuesta por Benzécri (1973) para representar y analizar gráficamente la relación que se establecen entre las variables categóricas (palabras y segmentos) definidas en la tabla de contingencia (Adriyanov, 2008; Fernández, 2002; Saavedra, 2012), en este caso, para mostrar la distribución de los perfiles léxicos de las filas y columnas de la 
matriz léxica a fin de identificar las asociaciones y similitudes existentes entre las palabras y segmentos textos del corpus (Hernández, 2012; Lebart, Salem \& Berry, 1998; Lebart, Morineau \& Piron, 2000).

f) El mapa perceptual. Representa el nivel de similitud y asociación entre las variables categóricas, a través de un conglomerado de puntos que se distribuyen es un espacio bidimensional según sus atributos, el cual, se determina a partir de la distancias entre ellas, de tal manera que, dos variables se consideran altamente relación cuando se encuentran próximas entre ellas y forma un ángulo igual o cercano a $0^{\circ}$ con respecto al origen del mapa, si es de $180^{\circ}$ entonces se consideran similarmente opuestos, y si se aproxima a $270^{\circ}$ o $90^{\circ}$, entonces puede decirse que tienen una baja relación (Caballero \& Vicente, 2011; Césari, 2002; Chue, Barreno \& Millones, 2007; IBOPE Media).

g) Análisis de las definiciones y resultados. Es un proceso deductivo desarrollado con la ayuda de un experto temático, capaz de inferir patrones de conocimiento significativos asociados con el conjunto de datos. En este caso, con los resultados del análisis unidimensional identifica las palabras claves con las cuales se establecen unas categorias de análisis preliminares. Posteriormente, con el análisis multidimensional establece las unidades temáticas emergentes inferidas y agrupadas según las similitudes de los segmentos de texto y las categorías previamente definidas. Finalmente, ambos resultados son utilizados para determinar una o más definiciones colectivas según las similitudes y diferencias conceptuales de los segmentos de texto.

\section{Resultados y discusión}

A partir del análisis lexicométrico se identificaron 457 palabras distintas del corpus de un total de 1109, de las cuales se seleccionaron 42 palabras cuya frecuencia era igual o mayor a tres, hasta crear una nube (Figura 1) con las palabras más representativas dentro del corpus.

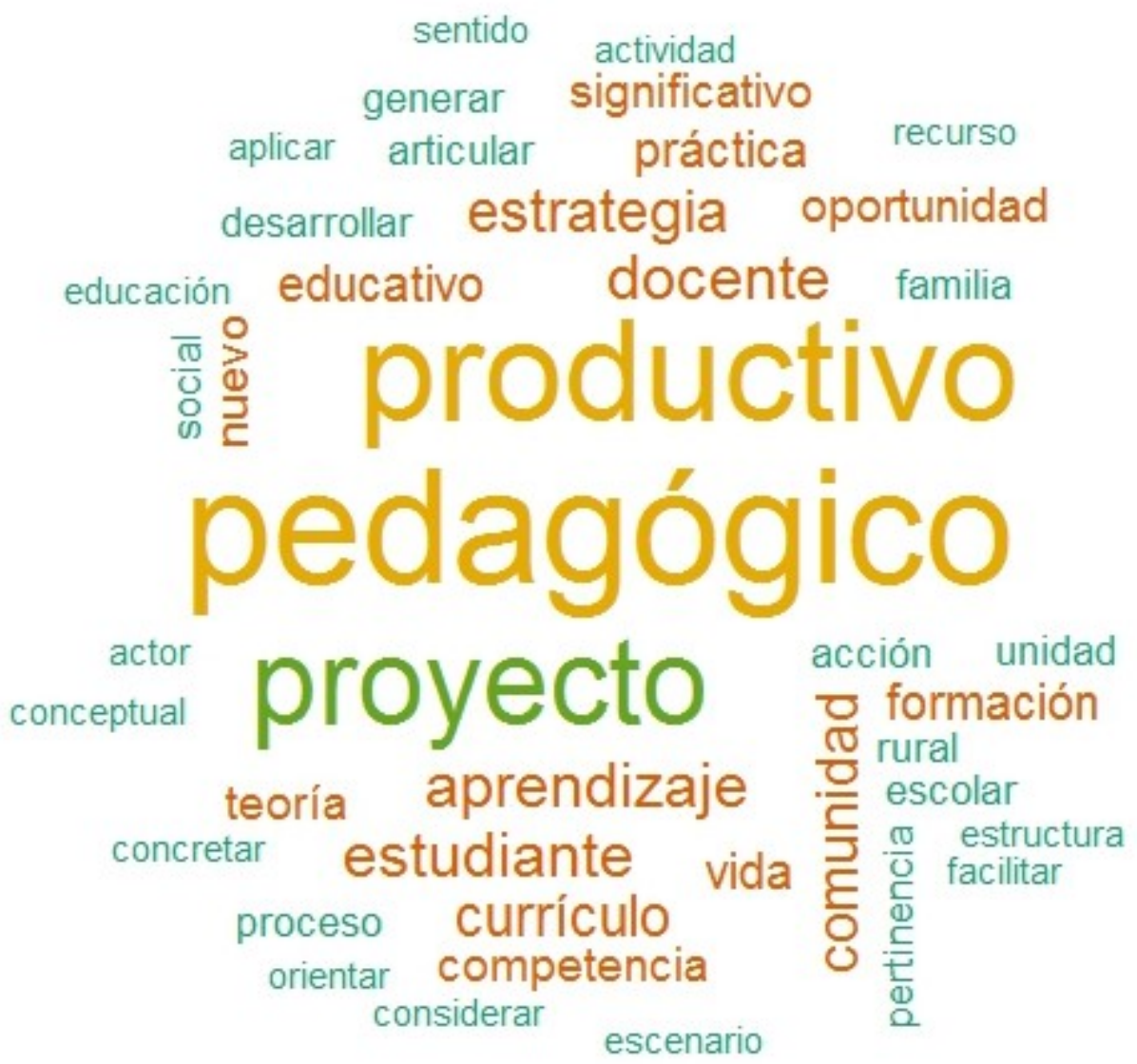

Figura 1 - Nubes de palabras más frecuentes. 
En la nube de palabras se pueden observar: proyecto y pedagógico como términos más relevantes dentro del primer nivel; seguida de productivo, docentes, estudiantes y aprendizaje en un segundo nivel; luego, estrategia en el tercero; comunidad y currículo en el cuarto; educativo, formación, práctica y vida en el quinto; nuevo, oportunidad, significativo y teoría en el sexto; acción, articular, desarrollar, escolar, familia, generar, pertinencia, proceso, rural, social y unidad en el séptimo; y finalmente, en el octavo, se identificaron actividad, actor, aplicar, conceptual, concretar, considerar, educación, escenario, estructura, facilitar, orientar, recurso y sentido.

Precisamente, con la lexicometría se construyen las categorías de análisis (Baudino \&Reising, 2000), las cuales, pueden inferirse del vocabulario, las palabras frecuentes y la nube de palabras inherentes al corpus. Todo esto basado en el análisis de los resultados por parte un experto temático quién se encarga de organizar y agrupar las palabras según las caracteristicas de los datos, sus conocimientos o estudios previos. En este caso, con las palabras: estudiantes, docentes, familia, comunidad y actor, se infiere la categoría Agentes Educativos. En las tres dimensiones definidas por Novoa (2005) y el MEN (2011) se incluyen las palabras: aprendizaje, estrategia, competencia, currículo, pedagógico, significativo, práctica, teoría y formación dentro de la Dimensión Pedagógica; proyecto, proceso, estructura, actividad y recursos en la Dimensión de Proyecto; y en la Dimensión Productiva, se agrupan: generar, nuevo, aplicar, concretar, desarrollar, productivo, unidad, y acción. 
Tabla 1 - Unidades temáticas emergentes del análisis lexicométrico y del análisis textual.

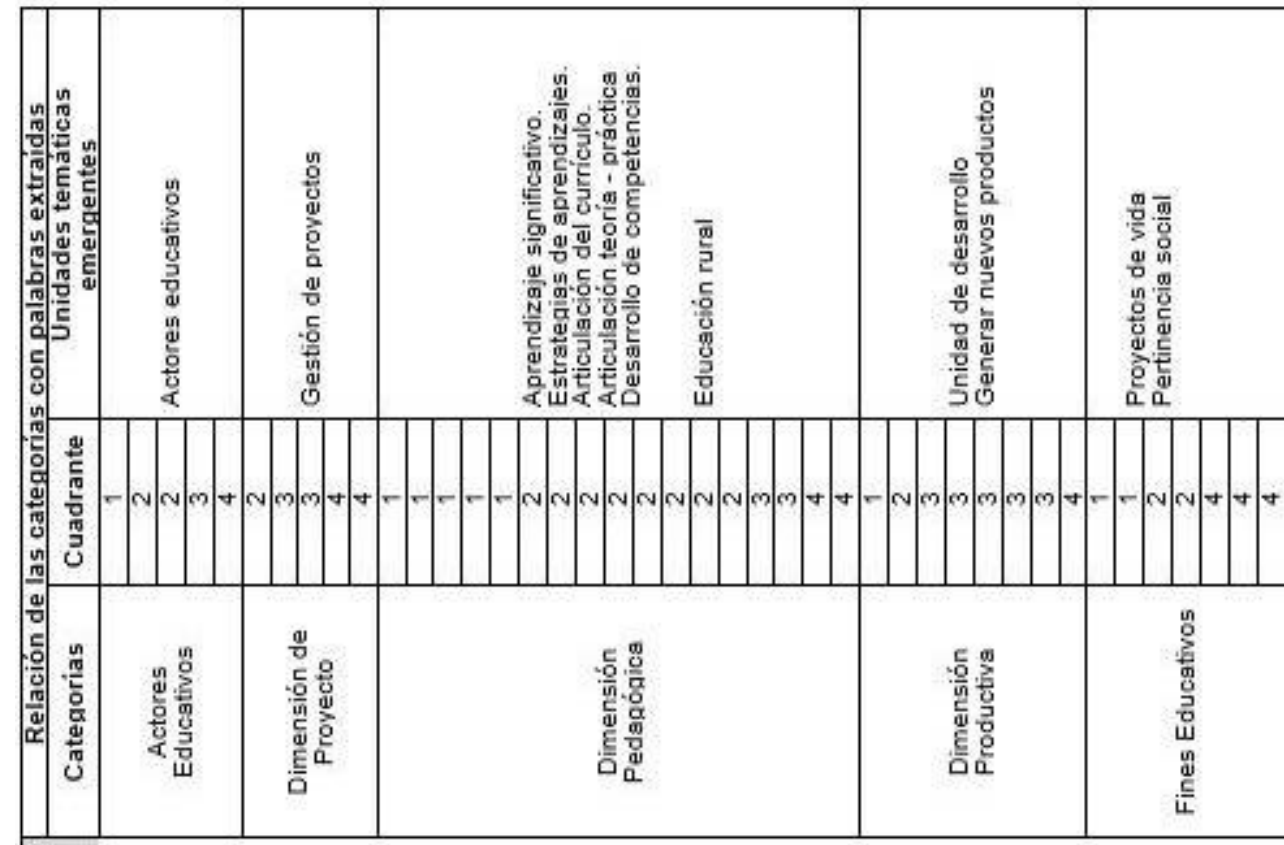

No-000-00-60000-0000m000-00000000-00-000000N

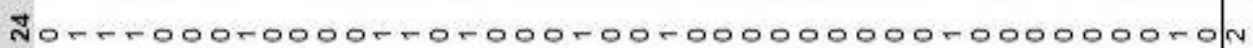
ñoo-00m-n-0-00000-00n-00000000000mo000-0000n

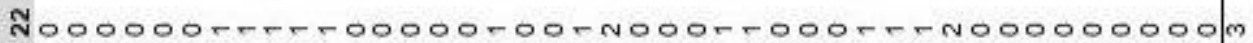
స̃ $000-N 00-0000000000-000-000-0-0-n=000-0-m$ กิ0000000-0000000000000000-0000000-000000000m $5000-500-00000000000-0000-0000000-50000000 \mathrm{~m}$ \% -0-0000-000-0000000-000-00000000-000N000-rt ₹0000000-000-00-0000-000000000000-0000-0000. ڤ00-0000-0000000NOr-0r000000000-0r-ro0000000m $\$ 0000-000-0-000000000000000-0000-0000-00000$ t Into m0000000-00-00000000N000000-00000m0-0000000m

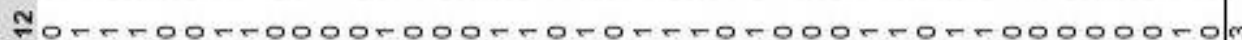
Fo0-0000000-0000N000--00000-00000-00000-000N

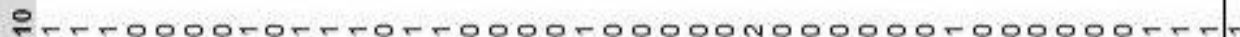
$00000000-00000000 N 00-0-000000000-700000000 \mathrm{~N}$

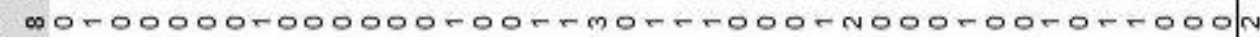

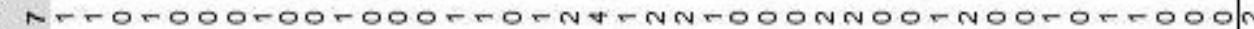

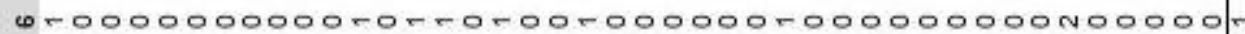

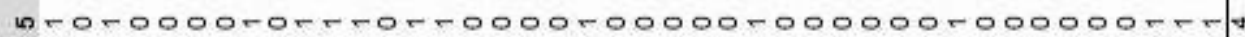
$\rightarrow 0 n-0000-00 N 000-000-n+-000000-0000000000000 N$ mo000000nO000-000000-000000000000-00000-000N

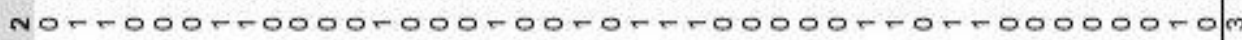
r-000000-00000000000-00000-000000N000000-0H 


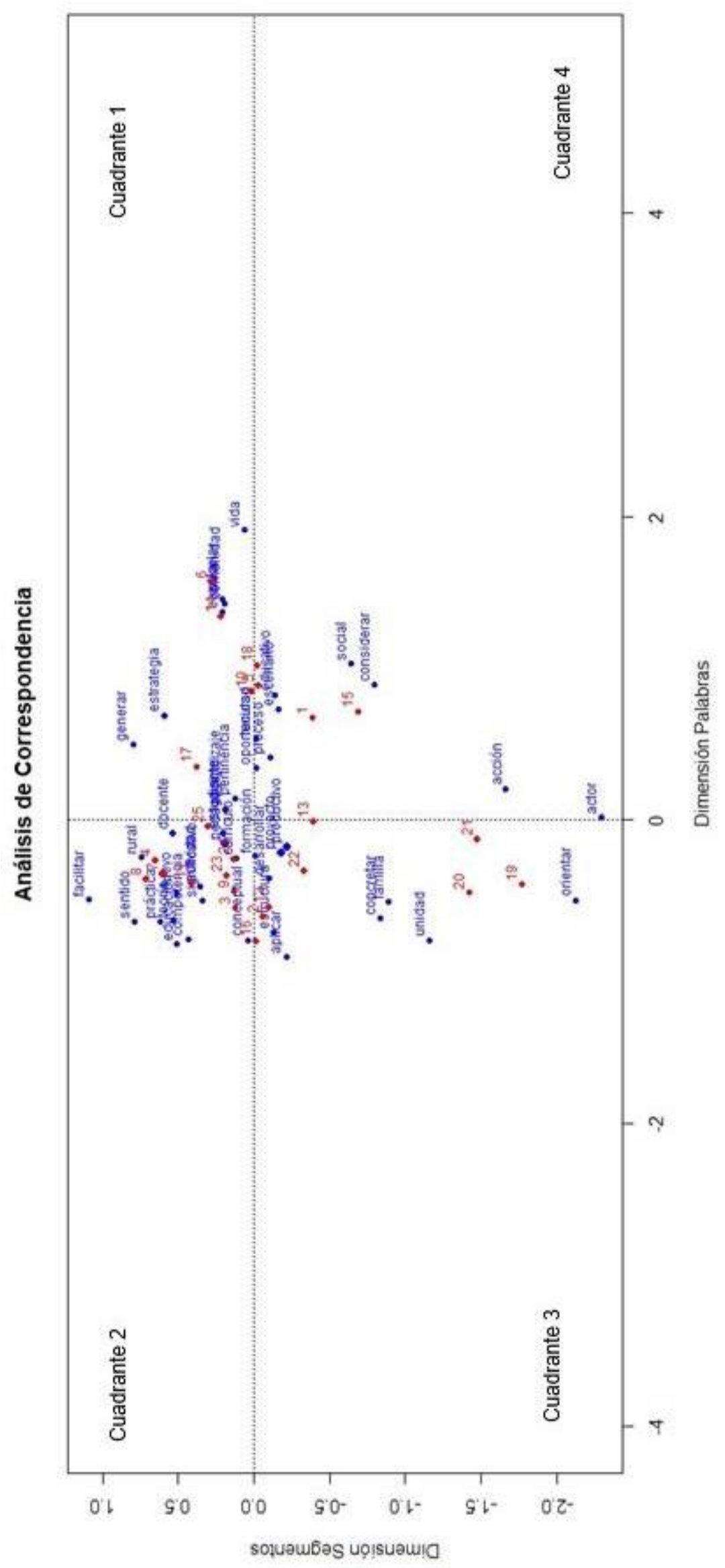

Figura -2- Mapa perceptual de las variables categóricas. 
Finalmente, se infiere la categoría Fines Educativos, que agrupa las palabras: pertinencia, vida, rural, sentido, considerar, oportunidad y social, las cuales, develan un entretejido semántico que va más allá del objeto de la dimensión pedagógica, debido a que éstas representan aspectos relacionados con los fines y propósitos esperados del proceso de formación, es decir, las opciones y posibilidades que brinda la escuela para que los jóvenes desarrollen sus proyectos de vida, así como, las capacidades para que se inserte en el mundo laboral, ofreciendo las oportunidades para que los sectores sociales y productivos se articulen a los procesos de formación, de tal manera que lo que se enseñanza y lo que se aprende, tenga sentido de pertinencia en el contexto que les rodea.

Después de obtener estos resultados, se aplicó el análisis descriptivo multidimensional para descomponer y comparar varias definiciones a fin de identificar las aproximaciones (distancias) y asociaciones (correlación) de sus segmentos y palabras, en este caso, se aplicó la técnica de análisis de correspondencia simple a la matriz léxica (Ver tabla 1), para identificar sobre el mapa perceptual las variables categóricas (Figura 2), las semejanzas y relaciones que existen entre los segmentos textos y las palabras. De esta manera, se extraen los patrones de conocimiento inherentes entre las distintas definiciones (segmentos de texto) para redefinir el concepto PPP desde el punto de vista de su representación colectiva.

Si comparamos los resultados de la Tabla 1 y la Figura 2, podemos identificar en el primer cuadrante (superior derecho) los segmentos de textos 6, 10, 14 y 17, quienes tienen proximidad con las palabras: vida, articular, comunidad, escolar, estrategia, generar, pertinencia y aprendizaje. Asimismo, podemos observar que los segmentos 6 y 14 se encuentran más próximos entre sí, lo cual quiere decir que sus definiciones guardan mayor semejanza en comparación con los otros. Además, estos hacen énfasis en el uso de las palabras: estrategia, comunidad, vida y escolar, con cierto grado correlación. Del mismo modo, emerge la unidad léxica vida, como una palabra con alto nivel de significancia y dispersión dentro del grupo, por tanto, puede proyectarse dentro de la categoría Fines Educativos como una unidad temática desde el punto de vista de los proyectos de vida.

Por otra parte, el segmento 10 se proyecta prácticamente sobre el perfil medio de las filas (palabras) del eje factorial de las $\mathrm{x}$, este posicionamiento sólo puede interpretarse como una definición que se caracteriza por utilizar un vocabulario común al grupo en general, especialmente, por el uso de las palabras articular, comunidad, estrategia y aprendizaje, todas éstas ubicadas en el primer cuadrante y cercanas al segundo cuadrante; destacándose la palabra aprendizaje, la cual se posiciona tanto en los perfiles medios de las columnas como en los perfiles medios de las filas, es decir que es una palabra representativa de uso colectivo, que puede utilizarse dentro del contexto de los PPP en dos sentidos, primero como una estrategia de aprendizaje y segundo como aprendizaje significativo, motivo por el cual, ambas han sido incluidas como unidades temáticas emergentes dentro de la categoría Dimensión Pedagógica.

En lo que respecta al segundo cuadrante (superior izquierdo), se identificaron las palabras: docente, estudiante, actividad, competencia, currículo, educación, facilitar, pedagógico, significativo, práctica, teoría, nuevo, rural y sentido, las cuales, se agrupan cercanas al centro de gravedad de ambos ejes factoriales y a los segmentos 3, 4, 7, 8, 9, 11, 23, 24 y 25. Estos resultados, pueden interpretarse como definiciones con mayores similitudes léxicas, todo a pesar de tener una correlación negativa con respecto a los segmentos del primer cuadrante, con quienes están poco o nada asociados, sin embargo, estos segmentos tienen un mayor número de palabras asociadas con la dimensión pedagógica, lo que significa que este grupo de definiciones enfatiza sobre los aspectos educativos en comparación con los segmentos de textos que utilizan palabras asociados con la dimensión de proyectos y productiva. Precisamente, con este conjunto de palabras se extraen las siguientes unidades temáticas: articulación curricular, desarrollo de competencias, articulación de la teoría y la práctica, y educación rural.

De manera complementaria, en el tercer cuadrante (inferior izquierdo) se identificaron los segmentos que tienen una correlación negativa con los del primer cuadrante, esto significa que sus proyecciones tienen un distanciamiento conceptual con los otros grupos, es decir, que son definiciones particulares que utilizan un léxico poco común, especialmente con los segmentos 19, 20 y 21 cuyo grado de proximidad están más cercana a las palabras productivo, proyecto, familia, unidad y orientar. Del mismo modo, las palabras concretar, familia y unidad están dispersas y distantes dentro del mapa. A diferencia de los segmentos 2, 12, 16 y 22 que tienen características similares con el subgrupo anterior, éstos se distinguen porque se encuentran ubicados muy próximos a los segmentos 
aglomerados en el segundo cuadrante y sobre los perfiles medios de las filas, lo cual quiere decir que, tienden a utilizar palabras habituales con respecto al grupo promedio. En este caso, este grupo utiliza las palabras: productivo, proyecto, formación, desarrollar, concretar, estructurar, unidad y aplicar; diferenciándose por su distanciamiento con la palabra unidad como la más representativa dentro del grupo. Con estos resultados, se identificaron unos patrones de conocimiento muy particulares alrededor de la participación de la familia como agente educativo y la estructura funcional de los PPP dentro de la dimensión productivo, de aquí que se consideren como emergente las siguientes unidades temáticas: gestión de proyectos, unidad de desarrollo y nuevos productos.

Finalmente, en el cuarto cuadrante (inferior derecho), se ubican los segmentos 1, 5, 15 y 18, los cuales, se caracterizan por tener pocas palabras cercanas, todo a pesar de compartir las palabras: social, educativo, oportunidad y considerar. De igual manera, estos segmentos se encuentran diametralmente opuestos a las definiciones del primer cuadrante, y distantes a las definiciones del segundo cuadrante, situación que puede interpretarse como diferencias conceptuales entre ellos, lo que permite identificar dos grandes grupos, los del primer cuadrante que se caracteriza por utilizar un vocabulario común entre la mayoría de los segmentos y los del tercer cuadrante, que tienden a utilizar un vocabulario especializado o poco común con descripciones opuestas a los argumentos utilizados por la mayoría de los autores, especialmente, si los comparamos con los segmentos 19 , 20 y 21 del tercer cuadrante, con los segmentos 1 y 15 del cuarto cuadrante; ambos grupos categorizados por utilizar un léxico muy especializado, pero, divergente en sus definiciones.

A su vez, las palabras actor y acción, son casos representativos que puede interpretarse como valores atípicos dentro del grupo, ya que, tienden alejarse del resto de las aglomeraciones, es decir, que son unidades léxicas muy especializadas o poco comunes. En vista de lo anterior, estos resultados son valorados desde el punto de vista de la identificación de las palabras con baja asociación frente al grupo promedio, sin embargo, en este análisis se considera su especificidad como un elemento diferenciador dentro del concepto PPP, especialmente la palabra social que ha sido incluida dentro de la unidad temática pertinencia social, por ser un aspecto destacado dentro del proceso de revisión documental del contexto histórico.

\section{Consideraciones finales}

Con la revisión documental y el análisis de los resultados, se identificaron cinco categorías conceptuales, las cuales se utilizaron para agrupar las palabras frecuentes de la matriz léxica, posibilitando la identificación de las unidades temáticas emergentes representadas en la Figura 3.
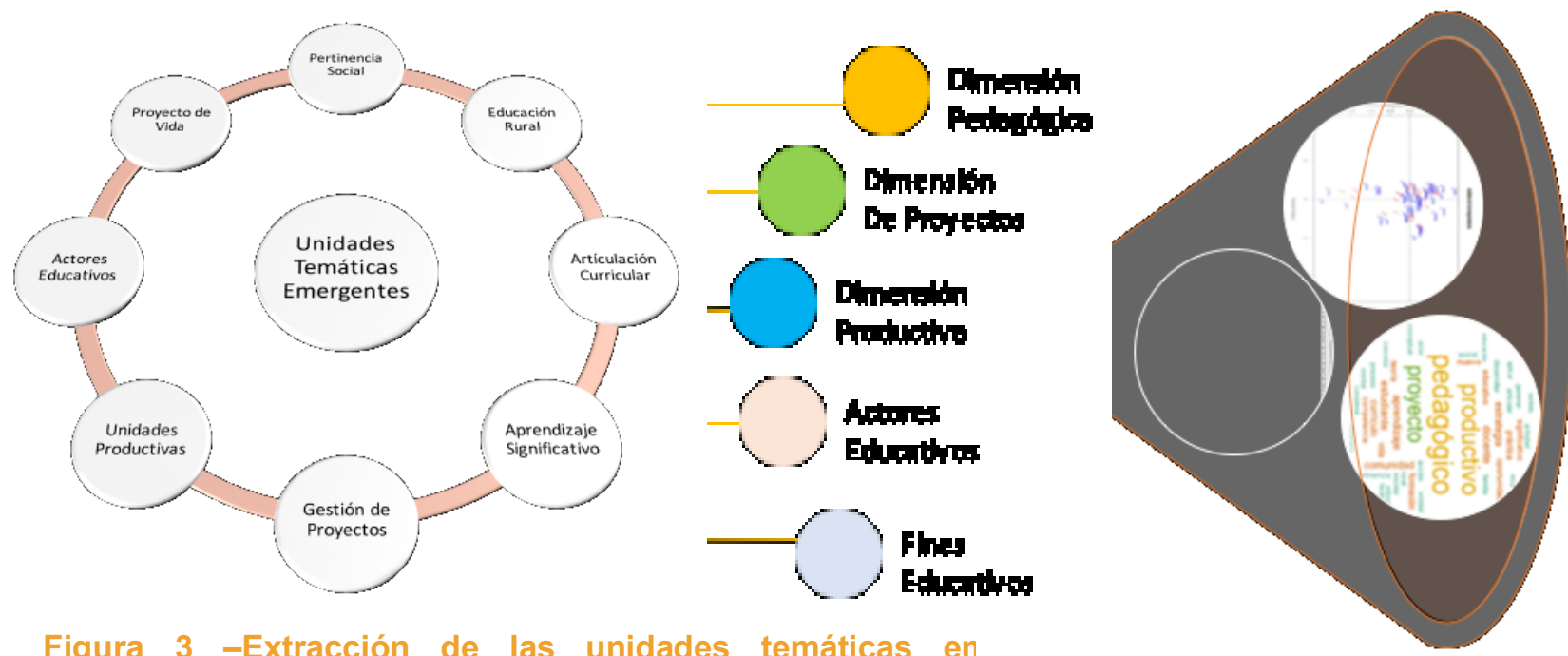

Figura 3 -Extracción de las unidades temáticas en investigaciones relacionadas con los PPP. 
Estas unidades son el resultado de la relación y asociación de las palabras frecuentes descritas en varias definiciones, por ende, pueden considerarse como una base de conocimiento confiable para fundamentar los PPP desde el punto de vista temático, asimismo, pueden ser utilizadas dentro del marco conceptual en futuras investigaciones relacionadas con este objeto de estudio, tales como: pertinencia social, educación rural, articulación curricular, aprendizaje significativo, gestión de proyectos, unidades productivas, actores educativos y proyecto de vida.

Con el análisis de las relaciones y asociaciones de las unidades léxicas y segmentos del primer cuadrante, se puede redefinir colectivamente el concepto PPP como estrategia de aprendizaje que articula la escuela con la comunidad, favoreciendo el desarrollo de proyecto de vida con pertinencia. Con los segmentos de texto que aparecen dentro del segundo cuadrante, se devela un patrón de unidades léxicas circunscritas dentro de la dimensión pedagógica, por tanto, éstos pueden utilizarse para hacer énfasis en los PPP como unidad pedagógica integrada al currículo, donde los estudiantes desarrollan competencias significativas y los docentes planifican sus actividades con sentido y pertinencia en el contexto rural, favoreciendo la articulación de la teoría y la práctica. Las unidades léxicas y temáticas emergentes del tercer cuadrante, se encuentran dentro de la dimensión de proyecto y la dimensión productiva, por tanto, una definición colectiva dentro de este grupo haría énfasis en la planeación de las unidades de desarrollo productivo, como en la participación de la familia. Por otra parte, los resultados del cuarto cuadrante son pocos representativos, sin embargo, de este grupo se destaca la pertinencia social como unidad temática que aporta valor al concepto desde el punto de vista de la categoría Fines Educativos.

Finalmente, cada uno de estos enfoques contribuye con la redefinición del concepto PPP, en la medida que no se pretende construir una definición apartada de los términos comunes sino de integrar los elementos más significativos que nutre la verdadera esencia pedagógica y social del mismo, es por ello que, este concepto debe al menos considerarse como estrategia de planeación educativa y articulación curricular utilizada por el docente para integrar los contenidos teóricos con experiencias prácticas, cuyo objetivo es potenciar en los estudiantes aprendizajes significativos y competencias básicas en función a la solución de problemas cotidianos, en contexto productivo, social, ambiental o cultural, donde la familia, la comunidad, la escuela y los sectores de producción participan activamente para viabilizar los proyectos de vida de los jóvenes, como una forma de desarrollo social y rural.

Con esto y todo lo anterior, se concluye que la lexicometría o el análisis de datos textuales es un enfoque cuantitativo que contribuyen a la extracción de conocimiento relevante para la redefinición de una expresión conceptual desde el punto de vista de su representación colectiva, sin embargo, para identificar las categorías de análisis este enfoque puede quedarse cortos sino se complementan con la revisión documental, a fin de extraer del legado histórico y del estado del arte, las unidades temáticas que sólo pueden emerger dentro de un análisis cualitativo realizado por un experto temático.

\section{Referencias}

Abasca, E., \& Franco, M.A. (2002). Análisis textual de encuesta: aplicación al estudio de las motivaciones de los estudiantes en la elección de su titulación. Metodología de encuesta, 4 (2), 195-209. Recuperado de https://goo.gl/WCWWB5.

Acosta, O.(2013). Extracción automática de relaciones léxico-semántica a partir de textos especializados (Tesis doctoral). Universidad Nacional Autónoma de México, México.

Baccalá, N., \& De la Cruz, M. (2000). La importancia de la estadística textual aplicada al estudio de las concepciones de enseñanza. En E. Brunet (Presidente), 5es Journées Internationales d'Analyse Statistique des Données Textuelles, Centro Regional Universitario Bariloche. Universidad Nacional del Comahue. Argentina. Argentina.

Barreto, I., Velandia-Morales, A., \& Rincón-Vásquez, J.M. (2011). Estrategias metodológicas para el análisis de datos textuales: aplicaciones en psicología del consumidor. Suma Psicológica, 18(2), 7-15. Recuperado de https://goo.gl/EQDJyG.

Baudino, V., \&Reising, A. (2000). Algunas reflexiones sobre el proceso de investigación desde la práctica. Cinta de Moebio. Revista de Epistemología de Ciencias Sociales, 9, 259-269. Recuperado de https://goo.gl/FWxY2O.

Borja-Orozco, H., Barreto, I., Sabucedo, J., \& López-López, W. (2008). Construcción del discurso deslegitimador del adversario: gobierno y paramilitarismo en Colombia. Universitas Psychologica, 7 (2), 571-583. Recuperado de https://goo.gl/VJ6BL8. 
Benzécri, J. (1973). L'Analyse des Données. Tomo II: L'Analyse des Correspondances. París: Dunod.

Bécue, M. (1991). Análisis estadístico de datos textuales: métodos de análisis y algoritmos. París: Cisia.

Bécue, M., Lebart, L., \& Rajadell, N. (1992). El análisis estadístico de datos textuales. La lectura según 10s escolares de enseñanza primaria. Anuario de Psicología, 55, 7-22. Recuperado de https://goo.gl/S397CU.

Bécue-Bertaut, M. (Diciembre de 2009). Mesurem els mots ? L ' anàlisi estadística de textos. En A. Ventura.(Presidencia), Jornades de Llengua i Estadística. Ponencia llevada a cabo en la de Lengua- Estadística de I'Idescat, Barcelona, España.

Caballero, D., \& Vicente, M. (2011). El HJ-Bitplot como herramienta en el análisis de grupo de discusión. (Trabajo de grado, Universidad de Salamanca). Recuperado de https://goo.gl/iPV8xF.

Campo, E. (2000). Análisis de la métrica en la aplicación de la estadística textual a la tipología de trayectorias. Revista Colombiana de Estadística, 23 (1), 1-13. Recuperado de https://goo.gl/KFHpv7.

Césari, M. (2007). Cartografiado de textos protocolo de exploración y visualización de datos textuales aplicados a la minería de textos (Tesis doctoral), Universidad Politécnica de Madrid, España. Recuperado de https://goo.gl/aebOfZ.

Césari, R. (2002). Métodos para investigación y formación de tesis. Recuperado de http://goo.gl/y1giY0.

Chue, J., Barreno, E., \& Millones, R. (2007). Sistema para el análisis estadístico de técnicas multivariadas del rendimiento académico de los estudiantes de una institución de enseñanza superior. Revista digital de la Facultad de Ingeniería de Sistemas, 2, 51-82. Recuperado de https://goo.gl/J3szrz.

Escalante, E. (2009). Métodos de análisis de las verbalizaciones: una contribución del análisis textual y análisis conceptual mediante el uso de software. Encuentro, 41 (83), 32-48. Recuperado de https://goo.gl/y6mlai.

Escobar, G. (2011).Proyectos pedagógicos productivos: Estrategias para el aprendizaje escolar y los proyecto de vida. En Gobernación del Valle del Cauca y Universidad del Valle. Estrategia y propuestas de educación pedagógica y rural: Nuevas opciones para la nueva ruralidad. Recuperado de https://goo.gl/phT1ee.

Fernández, F. (2002). El uso del análisis de correspondencia simple (ACS) como ayuda en la interpretación del dato en arqueología. Un caso de estudio. Boletín Antropológico, 55, 687- 713.Recuperado de https://goo.gl/9BBDsA.

Fernández, K., Modro-o, J., \& Landaluce, M. (2007). Exploración textual en el contexto del Modelo de Valores en Competencia. Aplicación al tipo de cultura de la UPV-EHU(1). Estadística Española, 49 (166), 501-530.

Gélvez, H. (2000). Conformación de la Comunidad Educativa.

Hernández, D. (2012). BibliomineR: una herramienta estadística para la revisión bibliográfica (Trabajo de Maestría), Universidad Politécnica de Cataluña, España. Adriyanov, B. (2010). Aportación del análisis canónico de correspondencias al análisis textual (Tesis doctoral), Universidad Politécnica de Catalunya. Recuperado de https://goo.gl/3iRFcw.

Lebart, L., Salem, A., \& Bécue, M. (2000). Análisis estadístico de datos y textos. España: Milenio.

Lebart, L., Morineau, A., \& Piron, M. (2000). Statistique exploratoire multidimensionnelle. Paris: Dunod.

Lebart, L., Salem A., \& Berry, E. (1998). Exploring Textual Data, Dordrecht: Kluwer. Academic Publishers. https://doi.org/10.1007/978-94-017-1525-6

Manual para el usuario de Correspondencia y Cluster Análisis. Colombia: IBOPE Media.

Ministerio de Educación Nacional. (2010). Proyectos pedagógicos productivo. Una estrategia para el aprendizaje y el proyecto de vida. Recuperado de https://goo.gl/DjPTNy.

Moscoloni, N. (2011). Las nubes de datos: métodos para analizar la complejidad. Argentina: Universidad Nacional de Rosario.

Novoa, A. (2004). Educación y Producción en el Desarrollo Rural una Innovación Metodológica en el Caso de Colombia. En I Foro- Taller "Alternativa para la educación rural en Venezuela". Tarabana, Venezuela.

Novoa, A. (2005). Formulación de proyectos pedagógicos productivos - módulo para el aprendizaje. Bogotá, Colombia: Oficina IICA en Colombia y Ministerio de Educación. Recuperado de https://goo.gl/9VfTjC.

Orozco, H., Barreto, I., \& Sánchez, V. (2008). Actitudes del vendedor ambulante de la localidad de Chapinero frente a sus condiciones laborales y políticas. Diversitas,4 (2), 279-290. Recuperado de https://goo.gl/4qn1tl.

Oliva, L., Hernández, M., \& Castro, C. (2012). Más que palabras nos dicen los adolescentes que desean migrar . Estudio estadístico de las respuestas a una pregunta. Revista de Psicología Social Aplicada, 1(1), 55-73. Recuperado de https://goo.gl/BO79pY. 
Páramo, M. (2010). Análisis cualitativo de discursos grupales asistido por programa de software textstat: valoración de su utilidad en la exploración y relación de significados. Liberabit, 14 (2), 141-151. Recuperado de https://goo.gl/riEFGD.

Ramírez, Á. (2007). Pedagogía para Aprendizaje Productivo. Proyecto Pedagógicos Productivos y Desarrollo de Competencias. Bogotá, Colombia: Eco Edición.

Ramón, J. (2010). Formación de Competencias para salir de la Pobreza en Modelos Educativos Rurales. Buenos Aires, Argentina: CLACSO

Rodríguez, S., \& Alessandrini, D. (2014). Aplicación de técnicas de Análisis Estadístico de Textos a una encuesta con preguntas abiertas: comparación de los resultados obtenidos con post-codificación (Trabajo de pregrado), Universidad de la Republica de Uruguay, Uruguay. Recuperado de https://goo.gl/Gzl4DA.

Romero-Pérez, I., Barrera, L \& Miranda, J. (2017a). Bases conceptuales de los proyectos pedagógicos productivos. En Aguilar A; Orozco, A \& Jiménez, M. (Coord). Educación, desarrollo y representaciones sociales: estudios interdisciplinarios del Caribe. (pp. 71-98). Barranquilla: Universidad Simón Bolívar.

Romero-Pérez, I., Miranda, J. \& Alarcón-Vásquez, Y. (2017b). Impacto de los proyectos pedagógicos productivos en Colombia En R. Mora. (Ed.), La Gestión curricular como investigación para el desarrollo y fortalecimiento de los procesos de formación en organizaciones educativas. (pp. 225-258). Barranquilla, Colombia: Editorial Universidad Simón Bolívar.

Saavedra, R. (2012). El análisis de correspondencias conjunto y múltiple ajustado (Trabajo de maestría), Pontificia Universidad Católica del Perú, Lima, Perú. Recuperado de https://goo.gl/gEDGM4.

Satriano, C. \& Moscoloni, N. (2000). Importancia del análisis textual como herramienta para el análisis del discurso. Cinta de Moebio. Revista de Epistemología de Ciencias Sociales,9, 287-306. Recuperado de https://goo.gl/UVTODZ.

Silva, M., Hernández, M., \& Sosa, A. (2010). Contribución del Análisis Estadístico Textual a la labor del tutor académico y el orientador educativo. Revista de Investigación Educativa, 11, 1-21. Recuperado de https://goo.gl/UVTODZ.

Terrazas, W. (2016). Análisis lexicométrico aplicado al estudio de las concepciones de aprendizaje. Educar em Revista, 61, 241-255.

https://doi.org/10.1590/0104-4060.44222

Vázquez-Cano, E., Mengual-Andrés, s., \& Roig-Vila, r. (2015). Análisis lexicométrico de la especificidad de la escritura digital del adolescente en WhatsApp. Revista de Lingüística Teórica y Aplicada, 1, 83-105.

https://doi.org/10.4067/S0718-48832015000100005

Zamora, J., \& Vallejos, R. (2012). Aprendiendo estadística con R. VIII festival internacional de matemática. Liberia: Costa Rica.

Notas. Los anexos se encuentran disponibles en el siguiente enlace: hittps://figshare.com/articles/Proyectos Pedag gicos Productivos/3420967 


\section{Datos de los autores}

\section{Ivón Romero-Pérez}

Ingeniera de Sistema. Magister en Educación. Profesora del Departamento de Ciencias Sociales y Humanas. Universidad Simón Bolívar, Barranquilla, Colombia.

ivromero@unisimonbolivar.edu.co

\section{Yolima Alarcón-Vásquez}

Psicologa. Doctora en Psicología. Profesora de la Facultad de Ciencias Jurídicas y Sociales. Universidad Simón Bolivar, Barranquilla, Colombia.

yalarcon1@unisimonbolivar.edu.co

\section{Rafael García-Jimenez}

Licenciado en matemática y física. Magister en estadística aplicada. Profesor del Departamento de Ciencias Básicas Universidad Simón Bolívar, Barranquilla, Colombia.

rgarcia1@unisimonbolivar.edu.co

Recibido - Received: 2017-05-07

Aceptado - Accepted: 2018-02-07

\section{(cc) EY}

This work is licensed under a Creative Commons Attribution 4.0

United States License.

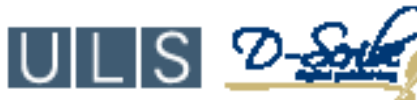

This journal is published by the University Library System of the University of Pittsburgh as part of its D-Scribe Digital Publishing Program and is cosponsored by the University of Pittsburgh Press. 